\title{
BIOMASA AÉREA, BIOMASA SUBTERRÁNEA Y NECROMASA EN UNA CRONOSECUENCIA DE BOSQUES TEMPLADOS CON APROVECHAMIENTO FORESTAL
}

\author{
Leopoldo Galicia ${ }^{1,4}$, Vinisa Saynes ${ }^{2}$ y Julio Campo 3 \\ 'Instituto de Geografía, Universidad Nacional Autónoma de México. México D.F., México \\ ${ }^{2}$ Colegio de Postgraduados, Campus Montecillo. Texcoco, Estado de México, México \\ ${ }^{3}$ Instituto de Ecología, Universidad Nacional Autónoma de México. México D.F., México \\ ${ }^{4}$ Autor para la correspondencia: Igalicia@igg.unam.mx
}

\begin{abstract}
Resumen: El aprovechamiento forestal sostenible ha sido considerado una estrategia para la adaptación y mitigación del incremento de $\mathrm{CO}_{2}$ atmosférico. En ese marco, los efectos de la extracción de madera en la biomasa y su recuperación en bosques templados de altura manejados están poco documentados. El objetivo de este estudio fue analizar las consecuencias de la extracción forestal en la biomasa y necromasa, y en su recuperación en bosques templados de la Sierra Norte de Oaxaca, México. Para ello se seleccionaron bosques con diferente tiempo de abandono luego del aprovechamiento ( 1 y 20 años de abandono), y bosques maduros en una vertiente seca $(900 \mathrm{~mm}$ de precipitación media anual) y en una húmeda (1,400 mm de precipitación media anual). En cada uno de los bosques se establecieron cinco parcelas $(10 \mathrm{~m} \times 50 \mathrm{~m})$ y se estimaron la biomasa aérea, la biomasa subterránea y la necromasa (residuos leñosos gruesos y mantillo). La contribución de estos compartimentos a la masa total de bosques aprovechados fue 74, 4, 17 y $5 \%$ por parte de la biomasa aérea, la biomasa subterránea, los residuos leñosos gruesos y el mantillo, respectivamente. El aprovechamiento redujo la magnitud y distribución de la biomasa y de la necromasa en los bosques luego de un año de la perturbación. Sin embargo, las masas y sus patrones de distribución presentaron recuperación luego de 20 años del aprovechamiento. La masa de los residuos leñosos gruesos aumentó $42 \%$ en los bosques luego de un año del aprovechamiento, por lo que es un componente que puede transformarse en un sumidero de $\mathrm{CO}_{2}$ atmosférico en el largo plazo.
\end{abstract}

Palabras clave: captura de carbono, México, piso forestal, residuos leñosos.

\begin{abstract}
The sustainable forestry has been considered a strategy for adaptation and mitigation of increased atmospheric $\mathrm{CO}_{2}$. In this context, the effects of timber harvesting on biomass and its recovery in highland managed temperate forests are poorly documented. The aim of this study was to analyze the impact of logging on biomass and necromass, and recovery in temperate forests of the Sierra Norte of Oaxaca, Mexico. For it, forest with different abandon time after harvest were selected (1 to 20 years of abandonment) and mature forests in a dry slope $(900 \mathrm{~mm}$ annual rainfall) and wet slope $(1,400 \mathrm{~mm}$ annual rainfall). In each of the forests five plots $(10 \mathrm{~m} \times 50 \mathrm{~m})$ were established and aboveground biomass, belowground biomass and necromass (coarse woody debris and litter) were estimated. The contribution of these compartments to the total mass of harvested forests was $74,4,17$ and $5 \%$ of the aboveground biomass, belowground biomass, coarse woody debris and litter, respectively. The harvest reduced the magnitude and distribution of biomass and forest necromass after a year of disturbance. However, the masses and their distribution patterns showed recovery after 20 years after harvesting. The mass of coarse woody debris increased $42 \%$ in the forest after a year after harvesting, it is a component that can become a sink for atmospheric $\mathrm{CO}_{2}$ in the longer term.
\end{abstract}

Keywords: carbon sequestration, Mexico, forest floor, coarse woody debris.

$\mathbf{L}$ os ecosistemas forestales son la principal fuente de madera y energía, como lo indican los 3,400 millones de metros cúbicos anuales de extracción de madera a nivel mundial registrada en 2005 (FAO, 2005); y al mismo tiempo son el almacén principal de $\mathrm{C}$ terrestre, ya que almacenan
$861 \mathrm{Pg} \mathrm{C}\left(1 \mathrm{Pg}=10^{15} \mathrm{~g}\right)$ (Pan et al., 2011). Este gran almacén de carbono está dividido en diferentes componentes, el $44 \%$ (383 Pg C) está en el suelo ( $1 \mathrm{~m}$ de profundidad), el $42 \%$ (363 Pg C) en la biomasa (aérea y subterránea), el $8 \%$ (73 Pg C) en la madera muerta y el $5 \%(43 \mathrm{Pg} \mathrm{C})$ en 
el mantillo, aunque esta distribución depende del tipo de bioma, la composición de especies y la intensidad de aprovechamiento (Pan et al., 2011). El Millennium Ecosystem Assessment Synthesis Report (2005) indica que la extracción de madera para pulpa, energía y papel se incrementó $60 \%$ en las últimas cuatro décadas y se realiza a tasas que no resultan sostenibles. Esta demanda creciente de recursos maderables ha contribuido al incremento de las emisiones de dióxido de carbono $\left(\mathrm{CO}_{2}\right)$ a la atmósfera, y ha reducido el potencial para la captura de carbono (C) aéreo y subterráneo de los ecosistemas forestales (Leighty et al., 2006; Newman y Hart, 2006; Roxburgh et al., 2006). La extracción forestal puede eliminar entre 30 y $90 \%$ de la biomasa de los ecosistemas forestales templados dependiendo de la intensidad de extracción de madera (Houghton, 2005). Burton et al. (2013) reportan que la biomasa arbórea contiene alrededor de $380 \mathrm{Mg} \mathrm{ha}^{-1}$, pero el manejo forestal reduce 100 y $156 \mathrm{Mg} \mathrm{ha}^{-1}$ de biomasa aérea con intensidad de extracción maderable alta y media, respectivamente. Balboa-Murias et al. (2006), Peichl y Arain (2007) y García-Gonzalo et al. (2007) también reportan la disminución inicial de la biomasa arbórea pero seguida de una recuperación después de 40 años de la perturbación. En los bosques templados se ha estimado que la biomasa de mantillo es entre 3.6 y $11.5 \mathrm{Mg} \mathrm{ha}^{-1}$; sin embargo, con el aprovechamiento de estos bosques se remueve hasta el $50 \%$ de esta biomasa (Currie y Nadelhoffer, 2002). La extracción de madera también reduce la biomasa radical $c a$. $50 \%$ en comparación con bosques sin aprovechamiento (Yin et al., 1989). Peichl y Arain (2007) encontraron que la contribución de la biomasa subterránea a la biomasa total disminuye en las primeras décadas posteriores al aprovechamiento forestal; sin embargo, es frecuente que su recuperación sea rápida (de 10 a 30 años; Laclau, 2003). En los bosques maduros los árboles muertos y la caída de ramas producen la acumulación de residuos leñosos gruesos (RLG) y debido a que pueden permanecer por decenas de años en el piso forestal frecuentemente son considerados como sumideros de C (Harmon y Hua, 1991). Sin embargo, en los ecosistemas bajo aprovechamiento la necromasa leñosa frecuentemente es modificada por las actividades de extracción de los árboles (Currie y Nadelhoffer, 2002). Se ha reportado que la extracción selectiva de árboles provocó un incremento de 16,14 y $7 \mathrm{Mg}$ de materia seca por hectárea de residuos leñosos en el piso de los bosques templados con extracción parcial, bosques sin extracción y bosques con extracción total, respectivamente (Brais et al., 2004). En contraste, también se han encontrado disminuciones iniciales en la necromasa leñosa después del aprovechamiento, seguida por la acumulación gradual de leños (Janisch y Harmon, 2002; Pregitzer y Eurskirchen, 2004; Peichl y Arain, 2006). A pesar de la importancia ecológica y económica que tienen estos bosques, las consecuencias de la extracción de madera en el potencial de captura de C, la estructura y distribución de biomasa en este ecosistema han sido escasamente documentadas.

La disponibilidad de agua puede regular la dinámica de los ecosistemas al afectar el balance entre la producción primaria y la descomposición, determinando el almacenamiento de $\mathrm{C}$ en los ecosistemas (Schlesinger, 1997). Modelos a escala regional han mostrado una relación lineal positiva entre la cantidad de lluvia y la producción primaria neta aérea y el C orgánico del suelo (Zhang et al., 2008). En los ecosistemas forestales el contenido máximo de mantillo y desechos leñosos frecuentemente se produce a niveles intermedios de lluvia, mientras que los valores más bajos ocurren en los extremos secos y húmedos del gradiente de lluvia (Austin, 2002). Meier y Leuschner (2010) encontraron que en los sitios más secos $(600 \mathrm{~mm}$ de precipitación media anual PMA) los almacenes de $\mathrm{C}$ en el mantillo, en el horizonte de fermentación y en el suelo mineral disminuyeron ( $c a .25 \%$ ) debido principalmente a que las tasas de descomposición fueron más altas que en los sitios más húmedos (970 mm de PMA). En contraste, también se ha reportado que sitios más secos tienden a capturar más $\mathrm{C}$ en el mantillo en comparación con aquellos más húmedos, donde la descomposición resultó ser más rápida (Kurz-Besson et al., 2006). Sin embargo, los efectos que producen las variaciones de la cantidad de lluvia sobre los almacenes de $\mathrm{C}$ en un mismo tipo de ecosistema son insuficientes ya que aún no se han podido determinar patrones consistentes.

En México los bosques templados contienen $c a$. 50 especies de pinos y 135 especies de encinos, lo cual representa más de la mitad del total de especies a escala mundial (Llorente-Bousquets y Ocegueda, 2008). Los bosques templados de México son el hábitat de las especies maderables con mayor valor comercial en el mercado forestal (FAO, 2006). Estos ecosistemas sufren una gran presión antrópica, al ser la extracción de madera un componente fundamental en la economía de las comunidades locales y, también en la economía nacional (Bray et al., 2008). El aprovechamiento forestal considera la aplicación de tratamientos silvícolas y tratamientos complementarios orientados a la producción de bienes en un bosque. El método de aprovechamiento más utilizado en México es el Método Mexicano de Ordenación de Bosques Irregulares (MMOBI) y promueve una estructura normal basada en la curva de Liocourt o " $J$ " invertida. En el país, las comunidades que poseen empresas forestales comunitarias frecuentemente practican la extracción selectiva y viven de los bosques, esforzándose por hacer un uso sostenible de los recursos forestales. Particularmente, en la Sierra Norte de Oaxaca los antecedentes de aprovechamiento alcanzan 20 años. A pesar de ello, el conocimiento respecto a las consecuencias que tiene el aprovechamiento en la magnitud y distribución de la biomasa, así como en la necromasa acumulada en el piso forestal es limitado. El objetivo de la presente investigación fue analizar el efecto histórico de la extracción forestal sobre la biomasa aérea y la 
subterránea, así como en necromasa de bosques templados bajo condiciones diferentes de régimen de precipitación.

\section{Materiales y métodos}

Sitios de estudio. La Sierra Norte de Oaxaca (SNO) en México ocupa 17,039 $\mathrm{km}^{2}$. Se localiza en la parte norte del estado, dentro de la región fisiográfica Sierras Orientales entre los 16.5 y $18.0^{\circ} \mathrm{N}$, y 95.1 y $96.9^{\circ} \mathrm{O}$ (Figura 1). La SNO está delimitada de acuerdo a la conformación de cuencas hidrográficas y compuestas por los sistemas montañosos de la Sierra Mixe y la Sierra de Juárez. Los suelos dominantes en la región son Acrisoles y Luvisoles en las partes más altas, y Litosoles hacia los piedemontes (INEGI, 2000). La temperatura media anual disminuye con la altitud de 22 a 12 ${ }^{\circ} \mathrm{C}$ en la vertiente húmeda, y de 24 a $16{ }^{\circ} \mathrm{C}$ en la vertiente seca. La PMA es de $900 \mathrm{~mm}$ en la vertiente seca, y de 1,400 mm en la vertiente húmeda (García, 1998).

La vegetación varía con la altitud y la orientación. En la ladera continental de la SNO existen bosques templados de pino, pino-encino y Juniperus distribuidos en el rango altitudinal entre 2,000 y 3,200 m. Los bosques de encino y de encino-pino ocupan las laderas de 1,400 a 2,000 m s.n.m. La SNO presenta un mosaico de tipos de vegetación con diferentes grados de vulnerabilidad al cambio climático y a la deforestación (Gómez-Mendoza et al., 2006). La SNO ha sido considerada como una región prioritaria para la conservación, constituyendo un corredor biológico entre la Chinantla y la región de los Chimalapas en Chiapas (Arriaga-Cabrera et al., 2009). A pesar de que la zona es reconocida por la práctica de aprovechamiento comunitario forestal sostenible, actualmente existe presión sobre las especies maderables, así como desmonte para prácticas agrícolas que producen pérdidas en la biodiversidad (Arriaga-Cabrera et $a l .$, 2009). Según el Resumen Público de Certificación de la
Comunidad Ixtlán de Juárez (SmartWood, 2001), el sistema de aprovechamiento es una combinación de diversas prácticas dirigidas a promover su sostenibilidad. La superficie total certificada es 15,749 ha, y la superficie de bosques destinada a la producción alcanza 6,687 ha (aproximadamente $42 \%$ del área certificada). Predominantemente se aplica el sistema de cortas intermedias mejor conocido como método de "árboles padres". Este método consiste en realizar aclareos de árboles jóvenes antes de que alcancen su turno comercial, con el fin de redistribuir el incremento y disminuir la competencia por luz y nutrientes para los árboles que se dejan en pie. El turno de corta es de 20 años, con ciclos de 10 años; $y$ al finalizar cada ciclo se elabora un nuevo programa forestal.

Diseño experimental. En cada condición climática: la vertiente seca (VS) y la vertiente húmeda $(\mathrm{VH})$ se seleccionaron bosques con aprovechamiento forestal histórico (1 y 20 años, y un bosque maduro). En cada vertiente se seleccionaron rodales con (1) bosque maduro, sin reporte de aprovechamiento forestal durante los últimos 60 años (BSM y BHM, para la VS y la VH, respectivamente); (2) bosque con un año de abandono después de la extracción forestal (BS1 y BH1), aprovechados en el año 2002 y (3) bosque con 20 años de abandono después de la extracción forestal (BS20 y BH20), aprovechado en el año 1983 (Tabla 1; Saynes et al., 2012).

Determinación de la biomasa aérea. En los bosques elegidos se establecieron cinco parcelas de $10 \times 50 \mathrm{~m}$ en junio de 2003. En cada parcela se determinó para cada árbol su especie y se midió su diámetro a la altura del pecho (DAP, $1.30 \mathrm{~m}$ altura) y su altura $(\mathrm{m})$. También se determinaron la densidad de árboles (tallos ha-1), el área basal $\left(\mathrm{m}^{2} \mathrm{ha}^{-1}\right)$ y la biomasa $\left(\mathrm{Mg} \mathrm{ha}^{-1}\right)$. La biomasa arbórea $\left(\mathrm{Mg} \mathrm{ha}^{-1}\right)$ se obtuvo
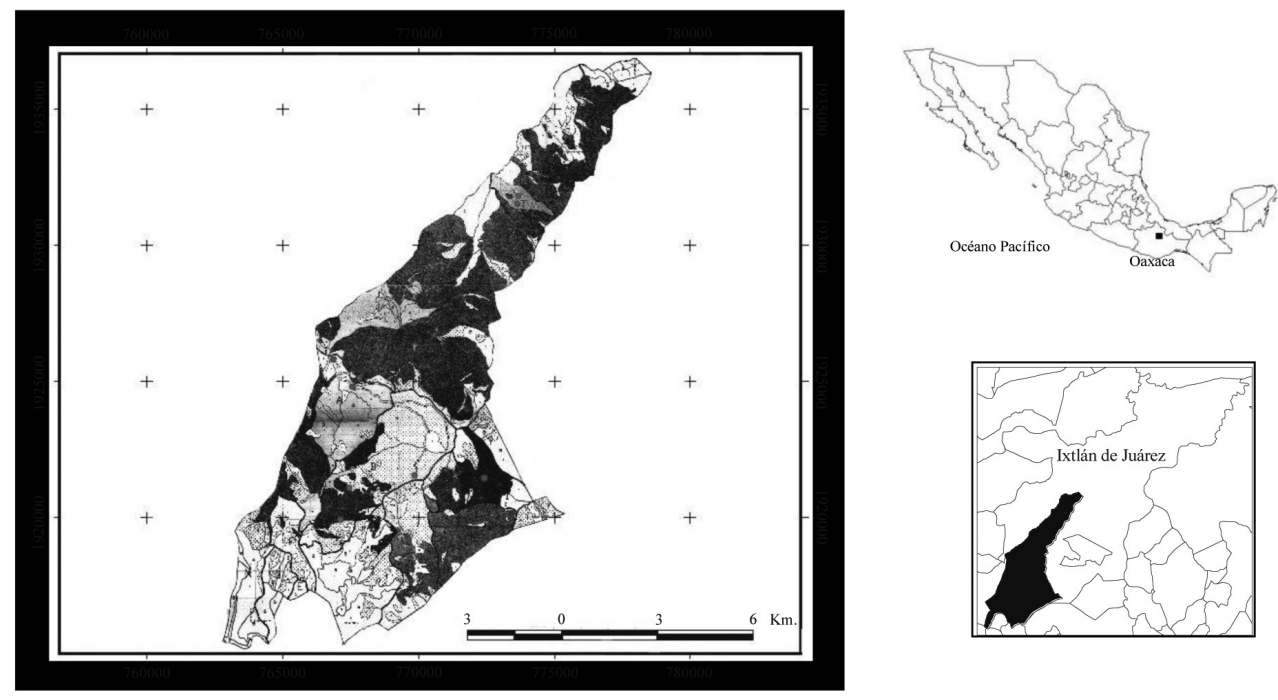

Figura 1. Localización de los bosques estudiados en la Sierra Norte de Oaxaca, México. 
Tabla 1. Características de los bosques templados estudiados en una vertiente húmeda y en una seca en Ixtlán de Juárez, Oaxaca. Los valores son medias y se indican entre paréntesis \pm 1 E.E.

*Para todos los árboles con diámetro a la altura del pecho $\geq 2.5 \mathrm{~cm}$, al inicio del estudio.

a Datos tomados de López (2005).

\begin{tabular}{|c|c|c|c|c|c|c|}
\hline & \multicolumn{3}{|c|}{$\begin{array}{l}\text { Región Seca } \\
\left(900 \mathrm{~mm} \mathrm{año}^{-1}\right)\end{array}$} & \multicolumn{3}{|c|}{$\begin{array}{l}\text { Región Húmeda } \\
\text { (1400 mm año-1) }\end{array}$} \\
\hline & $\begin{array}{c}\text { Bosque } \\
\text { Referencia } t\end{array}$ & 1 año & 20 años & $\begin{array}{l}\text { Bosque } \\
\text { Referencia }\end{array}$ & 1 año & 20 años \\
\hline \multicolumn{7}{|l|}{ Vegetación } \\
\hline *a Área Basal $\left(\mathrm{m}^{2} \mathrm{ha}^{-1}\right)$ & $19.0(1.8)$ & $24.6(3.0)$ & $4.5(0.6)$ & $15.0(1.4)$ & $5.7(0.8)$ & $4.7(0.4)$ \\
\hline aDensidad (individuos ha-1) & 1460 & 584 & 4192 & 1308 & 1656 & 2924 \\
\hline aAltura (m) & $11.3(0.5)$ & $14.4(0.9)$ & $7.3(0.2)$ & $9.7(0.4)$ & $8.5(0.4)$ & $9.6(0.2)$ \\
\hline \multicolumn{7}{|l|}{ Piso forestal } \\
\hline Residuos leñosos gruesos $\left(\mathrm{Mg} \mathrm{ha}^{-1}\right)$ & $79.31(4.71)$ & $177.26(7.51)$ & $63.00(2.85)$ & $95.54(6.85)$ & $150.71(6.67)$ & $120.06(5.87)$ \\
\hline Carbono en el mantillo (\%) & $49.74(0.41)$ & $47.77(1.85)$ & $50.52(0.23)$ & $49.13(0.60)$ & $49.85(0.62)$ & $50.17(0.14)$ \\
\hline \multicolumn{7}{|l|}{ Suelo } \\
\hline $\mathrm{pH}$ & $4.3(0.05)$ & $5.2(0.04)$ & $4.5(0.02)$ & $4.1(0.07)$ & $4.2(0.03)$ & $4.1(0.03)$ \\
\hline Densidad aparente $\left(\mathrm{g} \mathrm{cm}^{-3}\right)$ & $0.5(0.03)$ & $0.8(0.02)$ & $0.5(0.02)$ & $0.6(0.04)$ & $0.4(0.02)$ & $0.7(0.05)$ \\
\hline Carbono en el suelo 0-10 cm (\%) & $15.2(0.78)$ & $3.6(0.55)$ & $15.4(0.98)$ & $12.6(1.25)$ & $18.5(1.43)$ & $10.1(1.42)$ \\
\hline Carbono en el suelo $10-30 \mathrm{~cm}(\%)$ & $9.83(0.22)$ & $1.77(0.13)$ & $11.15(1.66)$ & $4.56(0.65)$ & $9.26(0.66)$ & $4.84(0.99)$ \\
\hline Nitrógeno en el suelo 0-10 cm (\%) & $0.61(0.0)$ & $0.57(0.0)$ & $0.67(0.0)$ & $0.45(0.0)$ & $0.53(0.0)$ & $0.58(0.0)$ \\
\hline Nitrógeno en el suelo $10-30$ cm (\%) & $0.57(0.0)$ & $0.59(0.0)$ & $0.69(0.0)$ & $0.47(0.0)$ & $0.56(0.0)$ & $0.59(0.0)$ \\
\hline
\end{tabular}

por medio de la utilización de ecuaciones alométricas a partir de las estructuras diamétricas (Tabla 2).

Determinación de la biomasa subterránea. Para determinar la biomasa subterránea (raíces finas) en cada parcela se obtuvieron dos muestras compuestas para cada una de las tres profundidades $(0-10,10-20$ y $20-30 \mathrm{~cm})$ en cada uno de los rodales ( $\mathrm{n}=180$ muestras). Para la determinación de la biomasa de raíces finas se colectó una muestra de suelo con un nucleador de $5 \mathrm{~cm}$ de diámetro y $10 \mathrm{~cm}$ de profundidad. Las raíces finas fueron consideradas como menores de $5 \mathrm{~mm}$ de diámetro y no se distinguieron entre vivas y muertas. Las muestras fueron transportadas al laboratorio donde se procedió a la extracción, lavado, secado y pesado de las raíces para la estimación de su biomasa.

Tabla 2. Ecuaciones alométricas para estimar la biomasa arbórea de las especies bajo aprovechamiento forestal en este estudio.

\begin{tabular}{lcc}
\hline Especie & Modelo & Autor \\
\hline $\begin{array}{l}\text { Pinus ayacahuite } \\
\text { P. montezumae }\end{array}$ & $\mathrm{Y}=0.084 \mathrm{DAP}^{2.475}$ & Ayala (1998) \\
$\begin{array}{l}\text { P. pseudostrobus } \\
\text { P. oaxacanaphart }\end{array}$ & & \\
Pinus patula & $\mathrm{Y}=\mathrm{Exp}(-1.8621+2.2768$ & $\begin{array}{c}\text { Castellanos et al., } \\
\text { In (DAP)) }\end{array}$ \\
Abies religiosa & $\mathrm{Y}=0.0754 \times$ DAP2.513 & Avendaño (2006) \\
\hline
\end{tabular}

Determinación de la necromasa. Se consideró como residuos leñosos gruesos (RLG) a todas aquellas piezas leñosas $\geq 2.5 \mathrm{~cm}$ de diámetro y $>50 \mathrm{~cm}$ de longitud, (sensu Harmon et al., 2001). En el centro de cada parcela se estableció una sub-parcela $(2 \times 5 \mathrm{~m})$ donde se midieron los diámetros de los dos extremos y la longitud de todos los RLG. Los diámetros correspondientes a cada pieza de RLG fueron utilizados para calcular su volumen. El volumen (v) de cada pieza leñosa se determinó según el método propuesto por Harmon et al. (1986), calculando $\mathrm{v}$ mediante la fórmula del cilindro (Rubino y McCarthy, 2003):

$$
V=\left[\pi h\left(R_{1}^{2}+R_{1} R_{2}+R_{2}^{2}\right)\right] / 3
$$

Donde $h$ es la longitud del leño y $R_{1}$ y $R_{2}$ el diámetro en cada extremo del leño.

Para obtener la densidad de los RLG se cortaron muestras cúbicas sólidas de $8 \mathrm{~cm}^{3}$ a cada una de las piezas leñosas. Se registró el peso de cada una de las submuestras (cubos de $8 \mathrm{~cm}^{3}$ ) para obtener la densidad. Las piezas en estado de descomposición avanzado frecuentemente pierden su forma cilíndrica y los tejidos leñosos son demasiado suaves para poder obtener las muestras cúbicas sólidas. En estos casos se colectó una porción de la pieza leñosa y se llenó un cubo de plástico con un volumen de $8 \mathrm{~cm}^{3}$. Para obtener el peso de cada pieza leñosa se utilizó la fórmula:

$$
\rho=\frac{m}{v}
$$


Donde $\rho$ es la densidad de la pieza leñosa; $m$ es la masa y $v$ es su volumen. Despejando la masa se tiene:

$$
m=\rho \times V
$$

En cada una de las cinco parcelas de $10 \times 50 \mathrm{~m}$ de cada bosque se colectó el mantillo en una sub-parcela $\left(1 \mathrm{~m}^{2}\right)$. En cada muestra de mantillo se discriminaron los siguientes componentes: hojas, acículas, madera (ramillas o pequeñas piezas leñosas $\leq 2.5 \mathrm{~cm}$ de diámetro y $\leq 50 \mathrm{~cm}$ de longitud), órganos sexuales (incluyendo flores y frutos) y material no identificado (fragmentos de los componentes anteriores que midieron entre 0 y $1 \mathrm{~cm}$ ). Cada fracción fue secada a $60^{\circ} \mathrm{C}$ durante $48 \mathrm{~h}$ y se determinó su peso. Finalmente se obtuvo la necromasa total del mantillo y de cada uno de sus componentes.

Análisis estadístico. La variación en la biomasa aérea, la masa de mantillo, las raíces y los residuos leñosos gruesos y sus componentes fueron explorados mediante el análisis de varianza de dos factores (región climática y edad del bosque). Cuando un factor fue significativo se realizaron comparaciones múltiples con la Prueba de Tukey $(P>0.05)$ corregida por Bonferroni $(P=0.05)$. Los análisis estadísti-
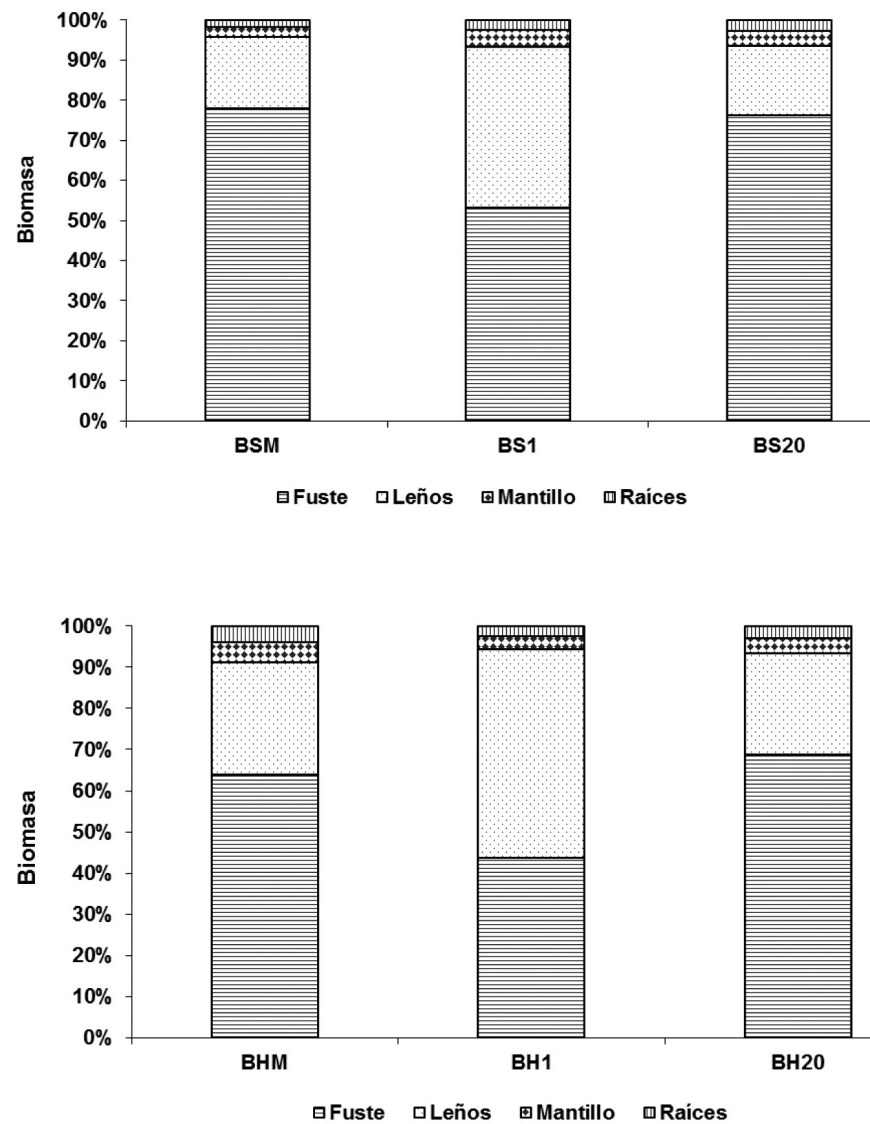

Figura 2. Biomasa arbórea, necromasa de leños y mantillo y biomasa de raíces $\left(\mathrm{Mg} \mathrm{ha}^{-1}\right)$ en una vertiente seca (A) y en una vertiente húmeda (B) de bosques templados de la Sierra Norte de Oaxaca, México. cos se realizaron con el paquete estadístico SPSS versión 10.0 para Windows.

\section{Resultados}

Biomasa aérea. En los bosques maduros la biomasa aérea arbórea total fue de $347 \mathrm{Mg} \mathrm{ha}^{-1}$ en el BSM y de $225 \mathrm{Mg}$ ha $^{-1}$ en el BHM (Figura 2A, B, respectivamente). La biomasa arbórea en los bosques sin aprovechamiento representó el $93 \%$ de la biomasa total (biomasa aérea y subterránea); y $70 \%$ incluyendo sólo la biomasa aérea y la necromasa (mantillo y RLG, respectivamente) de los bosques (Figura 2). El régimen de lluvia influyó en la biomasa arbórea $(F=$ 12.98, $P=0.001$ ). Los valores de biomasa aérea de los BSM y BS 1 fueron 1.5 y 1.8 veces más altos que su contraparte en la $\mathrm{VH}$, el caso contrario ocurrió en el $\mathrm{B} 2 \mathrm{OH}$.

Biomasa subterránea. La biomasa de raíces varió entre 2.2 y $9.8 \mathrm{Mg} \mathrm{ha}^{-1}$ (Figura 3). Esta biomasa representó $7 \%$ de la biomasa total en los bosques maduros y su contribución a la masa del suelo (p. ej.,necromasa del piso forestal) fue de $5 \%$. En los bosques maduros de la VS la biomasa subterránea fue 2.2 y $5.7 \mathrm{Mg} \mathrm{ha}^{-1}$ para las profundidades de $0-10$ y de 10$20 \mathrm{~cm}$, respectivamente (Figura 3A). En la VH, en el bosque sin aprovechamiento la biomasa osciló entre 2.3 y $9.1 \mathrm{Mg}$ $\mathrm{ha}^{-1}$ en la profundidad de $0-10$ y $10-20 \mathrm{~cm}$, respectivamente (Figura 3B). En la profundidad de $0-10 \mathrm{~cm}$ la biomasa de raíces fue $75 \%$ mayor comparada con la profundidad de $10-20 \mathrm{~cm}$, aunque esta diferencia no fue significativa estadísticamente.

El aprovechamiento forestal tuvo efectos en la biomasa de raíces únicamente en la profundidad de $0-10 \mathrm{~cm}$. La biomasa de raíces fue afectada significativamente por el aprovechamiento forestal $(F=15.2 ; P<0.001)$ y por la interacción entre la cantidad de lluvia media anual y el aprovechamiento $(F=32.7 ; P<0.001)$. En la VS la biomasa subterránea de $0-10 \mathrm{~cm}$ aumentó significativamente con el aprovechamiento forestal $(F=33.0, P<0.001)$; sin embargo este incremento sólo fue evidente en el BS1; en contraste en el BS20 se observó una biomasa similar a la correspondiente al BSM (Figura 3A). En la VH el aprovechamiento forestal también afectó significativo $(F=15.6, P=0.001)$, pero opuesto al observado en la VS (Figura 3A, B). En la $\mathrm{VH}$, el aprovechamiento redujo la biomasa de raíces en el $\mathrm{BH} 1$; sin embargo, luego de 20 años del aprovechamiento la biomasa de raíces se recuperó, siendo su magnitud similar a la observada en el BHM (Figura 3B).

Necromasa. La necromasa de RLG en los bosques estudiados varió de 63 a $177 \mathrm{Mg} \mathrm{ha}^{-1}$ (Figura 4). En los bosques maduros la necromasa de RLG fue de $79 \mathrm{Mg} \mathrm{ha}^{-1}$ en el BSM y de $95 \mathrm{Mg} \mathrm{ha}^{-1}$ en el BHM. El aprovechamiento forestal tuvo un efecto significativo sobre los RLG. En ambas vertientes la necromasa de RLG presentó un incremento signi- 

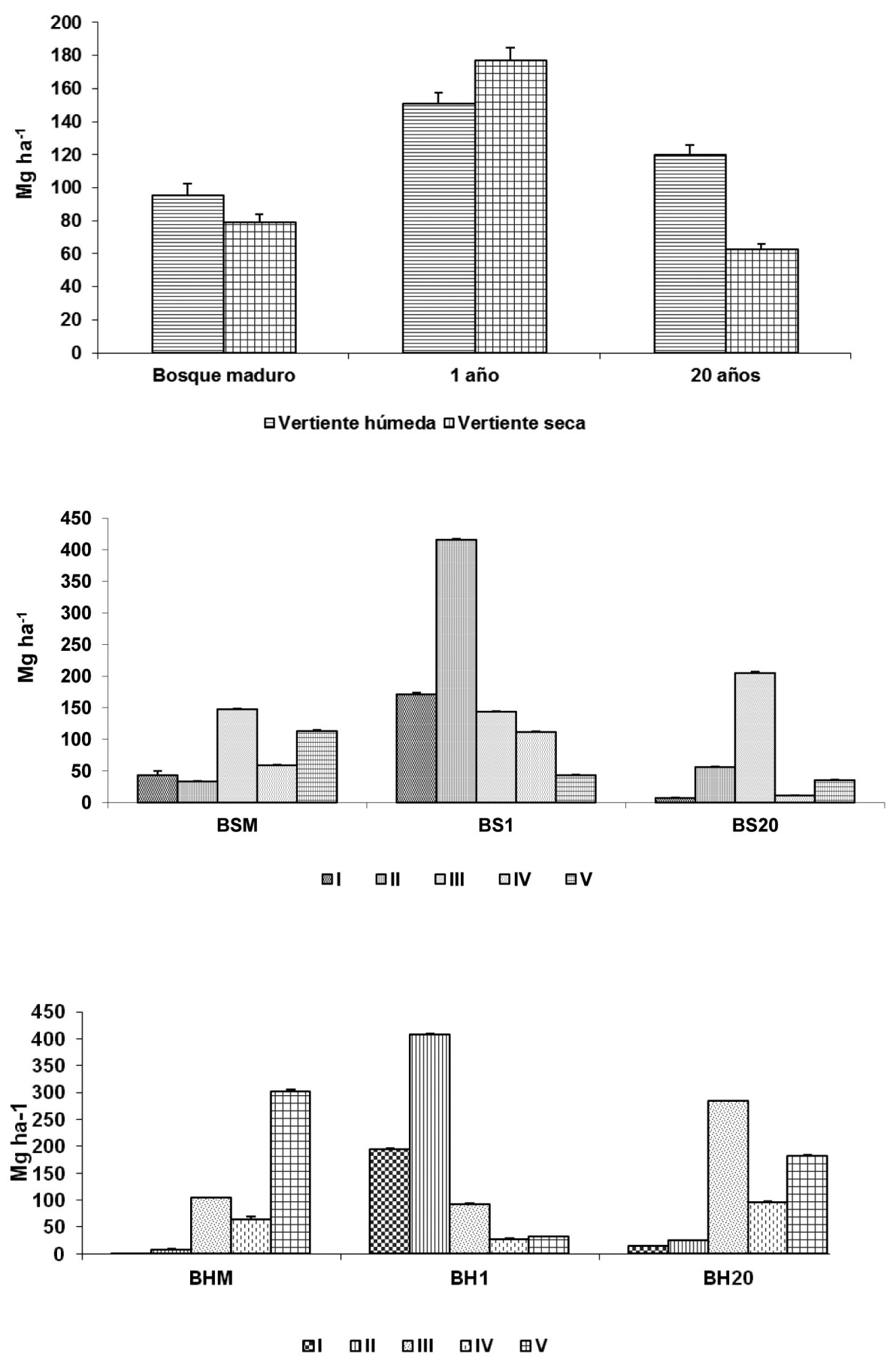

Figura 3. Biomasa de raíces $\left(\mathrm{Mg} \mathrm{ha}^{-1}\right)$ de 0-10 $\mathrm{cm}$ y de $10-20 \mathrm{~cm}$ de profundidad en una vertiente seca (A) y en una vertiente húmeda (B) de bosques templados de la Sierra Norte de Oaxaca, México.

ficativo $(F=17.47, P=0.001)$ después de un año del aprovechamiento. Sin embargo, transcurridos 20 años después de la extracción de árboles la necromasa de los RLG no fue distinta de aquella correspondiente a los bosques sin aprovechamiento (Figura 4).

La necromasa total de mantillo varió entre 8.7 y $17.9 \mathrm{Mg}$ ha $^{-1}$ (Figura 5A). En el BSM la necromasa de los bosques maduros fue de $10.6 \mathrm{Mg} \mathrm{ha}^{-1}$ y $17 \mathrm{Mg} \mathrm{ha}^{-1}$ en el BHM. El mantillo contribuyó con el $21 \%$ a la necromasa forestal (mantillo y RLG) y con el $5 \%$ considerando a la biomasa y a la necromasa. En las VS y VH los componentes con la mayor aportación a la masa de mantillo fueron las acículas y la madera (Figura 5B, C).

El aprovechamiento forestal afectó la acumulación de mantillo de forma diferente de acuerdo a la vertiente (interacción régimen de lluvia $\times$ tiempo de abandono: $F=32.5$, 

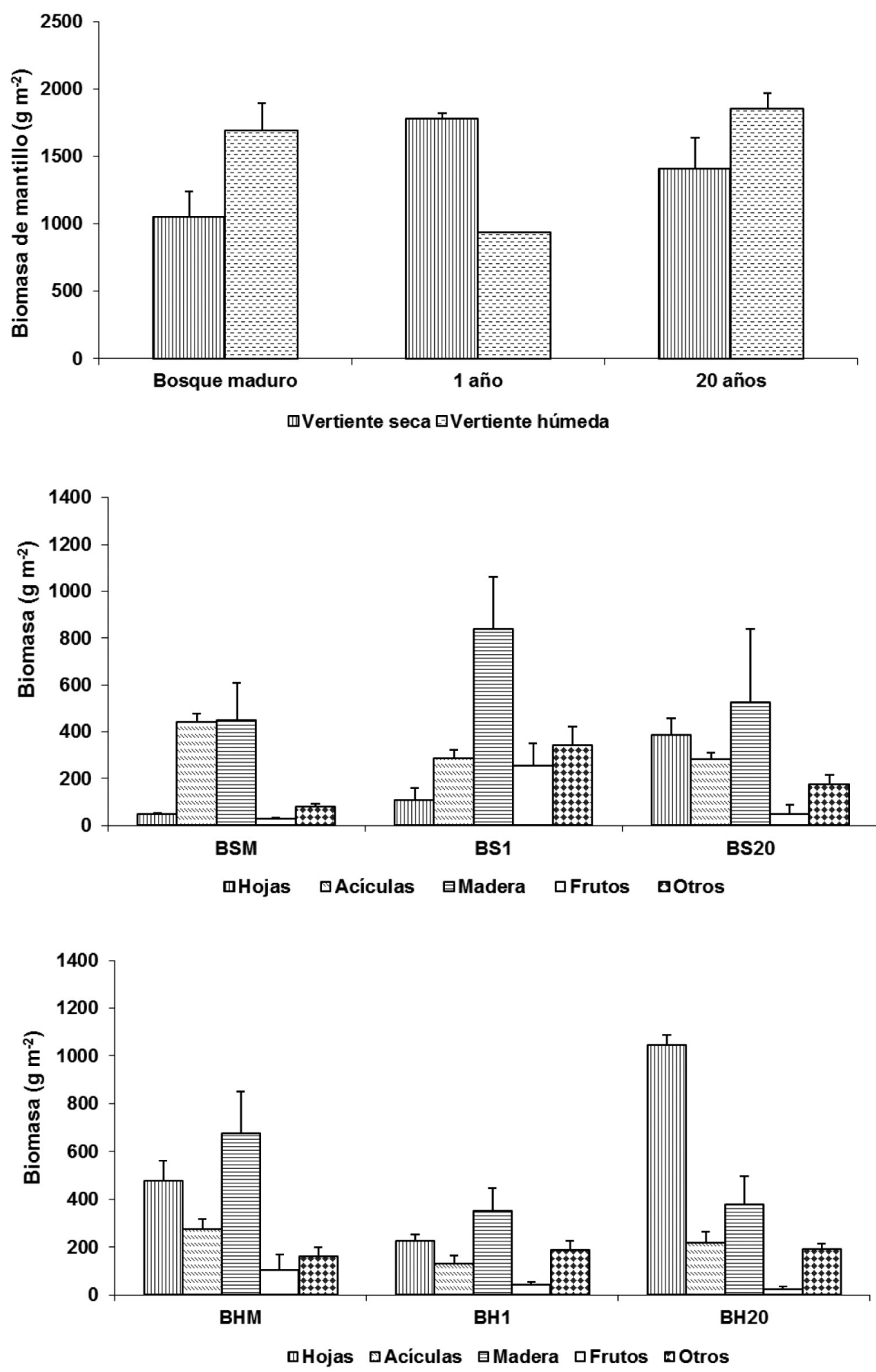

Figura 4. Necromasa de RLG $\left(\mathrm{Mg} \mathrm{ha}^{-1}\right)$ en una vertiente seca y en una vertiente húmeda de bosques templados de la Sierra Norte de Oaxaca, México. Las letras mayúsculas indican diferencias significativas $(P<0.05)$ entre vertientes seca y húmeda en un mismo bosque. Las letras minúsculas indican diferencias significativas $(P<0.05)$ del aprovechamiento forestal en una misma vertiente.

$P<0.001)$. En todos los bosques con aprovechamiento de la VS la masa de mantillo fue significativamente mayor a la correspondiente al BSM (Figura 5A). Sin embargo, en su contraparte húmeda (i.e., la $\mathrm{VH}$ ), la masa de mantillo se redujo en $\mathrm{BH} 1$, mientras que en el $\mathrm{BH} 20$ los valores medios fueron similares a los presentados por el BHM (Figura 5A). La extracción forestal también afectó la masa de hojas $(F=6.98, P<0.001)$, con efectos dependientes de la vertiente (interacción régimen de lluvia $\times$ tiempo de abandono) después del aprovechamiento $(F=3.89, P<0.001)$. En la VS, la masa de hojas incrementó en el BS20 en comparación con el BSM (Figura 5B). La extracción de madera también afectó la masa de acículas $(F=2.98, P=0.020)$. En la VS el BS1 y el BS20 fueron los sitios con mayor biomasa 

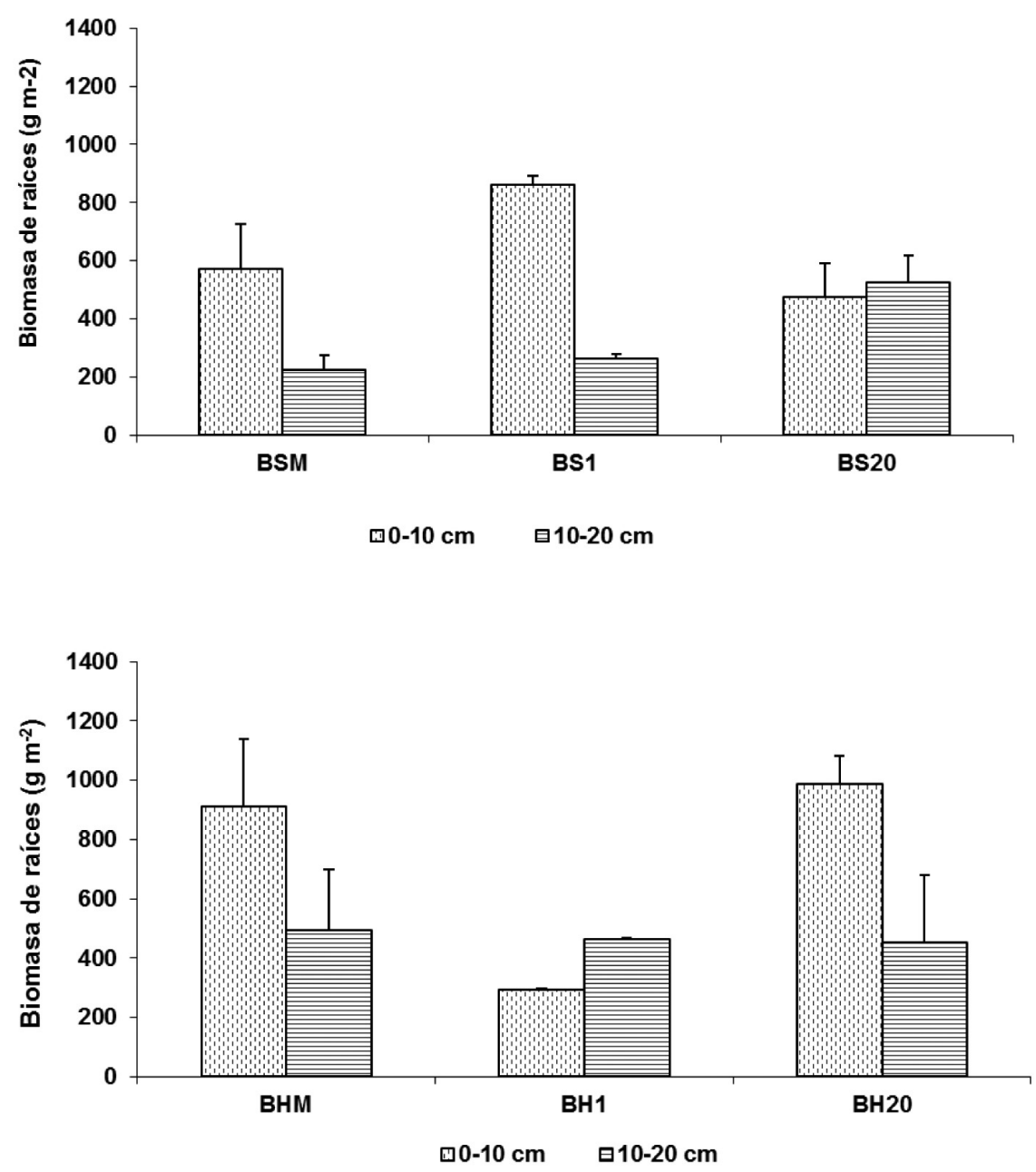

Figura 5. Necromasa total de mantillo (A) y de sus componentes $\left(\mathrm{Mg} \mathrm{ha}^{-1}\right)$ : necromasa de hojas, acículas, madera, frutos y otros en una vertiente seca (B) y en una vertiente húmeda (C) de bosques templados de la Sierra Norte de Oaxaca, México.

de acículas. En la VH la biomasa de acículas no varió significativamente entre las distintas edades de aprovechamiento (Figura 5C). El aprovechamiento forestal no afectó la acumulación de frutos en el piso forestal $(F=2.38, P=0.056)$, ni la acumulación del material no identificado (i.e., los fragmentos de hojas, acículas, madera, y frutos, de tamaños $\leq 1 \mathrm{~cm}$ ) (Figura 5C).

\section{Discusión}

Biomasa y necromasa: el factor aprovechamiento forestal. En el presente estudio se evidenció la mayor sensibilidad de los compartimentos de la biomasa aérea y de la necromasa de los bosques templados al aprovechamiento después de 1 y 20 años de la explotación. La biomasa arbórea de los bosques maduros y aprovechados en la comunidad de Ixtlán de Juárez varía entre 141 y $347 \mathrm{Mg} \mathrm{ha}^{-1}$; éstos rangos y magnitudes son comparables a los reportados para otros bosques templados (100 y $380 \mathrm{Mg} \mathrm{ha}^{-1}$; Tateno et al., 2004; White et al., 2004; Park et al., 2008). La biomasa arbórea de los bosques maduros en ambas vertientes fue mayor a la observada en los bosques con menos tiempo de abandono después del aprovechamiento ( $p . e j$., bosque de 1 año), como consecuencia del "adelgazamiento" en la densidad de árboles ya que generalmente los fustes contribuyen con el 70 a $95 \%$ a la biomasa aérea (Figura 2). Por otra parte, el ecosistema demostró una buena capacidad de resiliencia al recuperar sus valores de biomasa solo 19 años después, si se tiene en cuenta que ecosistemas similares tardaron el doble de tiempo en alcanzar la acumulación de biomasa previa al aprovechamiento (Porté et al., 2002; Balboa-Murias et al., 2006; Peichl y Arain, 2007 y García-Gonzalo et al., 2007). La rápida recuperación de la biomasa del bosque podría ser consecuencia de la baja extracción arbórea (sólo $25,000 \mathrm{~m}^{3}$ año-1), que podría favorecer el incremento diamétrico; es decir, que la extracción arbórea selectiva mejora las condiciones de crecimiento para los árboles remanentes, y por lo tanto la recuperación de la biomasa aérea (SmartWood, 2001).

En numerosos estudios respecto al aprovechamiento no se han considerado los RLG. En la SNO, los RLG representa- 
ron entre 77 y $177 \mathrm{Mg} \mathrm{ha}^{-1}$ en la VS, y entre 95 y $150 \mathrm{Mg}$ ha $^{-1}$ en la VH (Figura 3). Las necromasas en el piso forestal de los bosques de la SNO superan las magnitudes reportadas para bosques templados de Norteamérica (de 71-104 Mg ha-1; Gough et al., 2007). Harmon et al. (2001) estimaron que la necromasa de RLG contribuye con $c a .42 \%$ a la biomasa y necromasa total aérea. En nuestros sitios de estudio los RLG representaron aproximadamente el $17 \%$ de la biomasa y necromasa total aérea en condiciones naturales (p. ej., sin aprovechamiento conocido), pero esta incremento a $60 \%$ en los bosques bajo aprovechamiento. Está bien documentado que la masa de residuos leñosos varía con el estado sucesional del bosque, la senescencia y la existencia de perturbaciones naturales y antrópicas (Harmon et al., 1986; Carmona et al., 2002; Janish y Harmon, 2002). En la SNO, de forma consistente, la magnitud de la necromasa de RLG fue mayor en los bosques luego de un año de abandono en ambas vertientes. Nuestros resultados sugieren que en la SNO el factor que regula la magnitud de la necromasa de RLG es el aprovechamiento, favoreciendo la acumulación de hojas, ramas, y troncos en el piso forestal como lo reflejan los datos de las parcelas luego de un año de la extracción de árboles. La reducción del compartimento luego de 20 años es consecuencia del retiro y de la quema intencional de RLGs, así como de la descomposición de acículas (Cárdenas-Camargo, 2008).

La mayor parte de la producción primaria neta sigue el ciclo descomponedor sobre el piso forestal (Berg y Laskowski, 2006; Campo et al., 2014). En los bosques templados de la SNO la biomasa de mantillo varió entre 10.2 y 17.4 $\mathrm{Mg} \mathrm{ha}^{-1}$ en la VS, y entre 8.2 y $17 \mathrm{Mg} \mathrm{ha}^{-1}$ en la VH (Figura 4A). Estos valores se ubican en la parte media inferior del rango reportado para otros bosques templados de México (6.4 a $32 \mathrm{Mg} \mathrm{ha}^{-1}$; Díaz-Franco et al., 2007). A pesar de ello, confirman que en el mantillo hay un almacén considerable de $\mathrm{C}$ que puede ser alterado como consecuencia del aprovechamiento. No fue posible determinar un único efecto de la extracción forestal o del régimen de precipitación en éste almacenamiento de C en la SNO. Así, si bien en la VH la mayor masa de mantillo se observó en los bosques maduros de 1 y de 20 años, en su contraparte seca la masa de mantillo fue mayor en el bosque de 1 año. Ha sido documentado que esta acumulación de mantillo (p. ej., en el bosque de un año) puede representar un importante almacén de $\mathrm{C}$ y de nutrientes para sostener la demanda de bioelementos por parte de los bosques secundarios bajo aprovechamiento intensivo (Laiho et al., 2003).

La biomasa subterránea representa hasta $20 \%$ de la biomasa total en los bosques templados (Xiao y Ceulemans, 2004; Peichl y Arain, 2007). En la SNO la biomasa subterránea contribuyó entre el 1.3 y $3.7 \%$ a la biomasa total; es decir, es menor en comparación con lo reportado en otras investigaciones en bosques templados, probablemente porque en nuestra investigación sólo se consideraron las raíces finas. Por otra parte, la biomasa subterránea se concentró en los primeros $10 \mathrm{~cm}$ del perfil del suelo, en una proporción de 2 a 1 en todos los bosques (Figura 5). Esta relación de distribución de las raíces en el perfil del suelo resultó similar a la reportada por Yin et al. (1989) y por Tufekcioglu et al. (2003), sugiriendo un ecosistema limitado por recursos nutricionales y/o hídricos, dada importante asignación de $\mathrm{C}$ a la parte superficial del suelo. Esta biomasa demostró ser sensible al aprovechamiento, la cual redujo sus valores en un $45 \%$, magnitud similar a otros estudios. Se ha reportado que la extracción selectiva reduce la biomasa radicular ca. $50 \%$ en comparación con bosques sin aprovechamiento (Yin et al., 1989; Tang et al., 2005), sin embargo, es frecuente que su recuperación sea rápida (10-30 años); lo que indica una asignación importante de $\mathrm{C}$ en un período relativamente corto (Laclau, 2003). De hecho, en los bosques con aprovechamiento existe una rápida recuperación de la biomasa de raíces finas. Yin et al. (1989) encontraron que la biomasa de raíces finas es mayor en bosques de Quercus que fueron aprovechados $\left(12 \mathrm{Mg} \mathrm{ha}^{-1}\right)$ en comparación con bosques maduros $\left(7 \mathrm{Mg} \mathrm{ha}^{-1}\right)$ y bosques no aprovechados (6 $\left.\mathrm{Mg} \mathrm{ha}^{-1}\right)$. La contribución de la biomasa subterránea a la biomasa total disminuye en las primeras décadas posteriores al aprovechamiento forestal; sin embargo, esta contribución permanece constante en la fase de maduración de los bosques (Peichl y Arain, 2007).

Considerados los diferentes compartimentos de forma conjunta, permiten reconocer que éstas prácticas forestales en la SNO favorecen la recuperación de la biomasa en solo 20 años. Sin embargo, cabe mencionar que para ello resulta imprescindible la a minimización de las alteraciones del sitio mediante una tala de impacto reducido, así como no remover los RLG, los cuales permiten mantener un almacenamiento de $\mathrm{C}$ importante, contribuyen a la reducción de la erosión (no evaluada en éste estudio), y representan una fuente de recursos nutricionales que favorecen la recuperación del ecosistema forestal en términos de almacenamiento de masa y con ello de captura de C.

Biomasa y necromasa: el factor régimen de lluvia. La variación natural en la cantidad de precipitación permitió evaluar los efectos del régimen de lluvia en la biomasa y necromasa de los bosques templados naturales y aprovechados. GarcíaGonzalo et al. (2007) encontraron que un ambiente más cálido y húmedo produce un incremento en la biomasa arbórea y en los almacenes de C. En los bosques maduros de la SNO localizados en la VS la magnitud de su biomasa aérea superó a la correspondiente a los bosques de su contraparte húmeda, pero este patrón cambió después de 20 años del aprovechamiento. Se ha documentado que el aprovechamiento y las variaciones climáticas pueden oscurecer los efectos de la edad de los bosques. Los bosques jóvenes presentan más cambios en el microclima del suelo y a nivel del dosel (Gough et al., 2005). El aprovechamiento frecuentemente disminuye la densidad del dosel, y aumenta la evapotranspi- 
ración y la temperatura del suelo como resultado del incremento de áreas expuestos a la radiación solar directa (Pypker y Fredeen, 2003; Tang et al., 2005). Si bien la VS presentó una biomasa inicial mayor que la correspondiente a la VH, y por tanto una mayor acumulación de $\mathrm{C}$, nuestros resultados indican también su mayor vulnerabilidad, reflejando el efecto de la disponibilidad de agua en las tasas de los procesos ecosistémicos, evidenciado en el BS20 donde la magnitud de la biomasa arbórea disminuyó de forma significativa. Los efectos del aprovechamiento en la biomasa subterránea también demostraron ser dependientes del régimen de lluvia. El incremento de la biomasa de raíces en los bosques con menor tiempo de abandono desde el aprovechamiento (un año) con respecto a los bosques maduros y de 20 años en la VS pudo deberse a que la extracción modificó el microclima del suelo (temperatura y humedad edáficas) favoreciendo la producción subterránea. Por el contrario, en la VH la mayor acumulación de biomasa de raíces ocurrió en los bosques maduros y luego de 20 años del aprovechamiento, en comparación con los bosques de aprovechamiento más reciente (un año), debido probablemente a una asignación mayor de $\mathrm{C}$ a las partes aéreas.

Implicaciones para la acumulación de C. Los resultados del presente trabajo sugieren que actualmente los bosques en la SNO están acumulando biomasa. Entre los ecosistemas terrestres las especies de crecimiento rápido, especialmente las coníferas son almacenes de C importantes (Gucinski et $a l ., 1995)$, y pueden contribuir a la mitigación del cambio climático global porque son árboles altamente eficientes en términos de captura de C (Laclau, 2003). Esta eficiencia de las coníferas son relevantes en bosques como los de la SNO que se caracterizan por una distribución de edades desigual con dominancia de bosques jóvenes y de crecimiento rápido, y probablemente continuarán incrementando su biomasa en los próximos 20 años (Lindner y Karjalainen, 2007). En los bosques sin aprovechamiento la necromasa (mantillo y RLG) contribuyó con ca. $18 \%$ de la masa total de estos bosques; sin embargo, luego de un año de la extracción de los árboles la necromasa forestal representó $c a .60 \%$ de la masa forestal, lo que indica que después del aprovechamiento una gran parte de la biomasa aérea termina en el piso forestal. De hecho, se estimó una necromasa de RLG alta (hasta de $177 \mathrm{Mg}^{1}$ ), la magnitud de esta necromasa indica que es un componente estructural masivo de los bosques manejados, y que tienen el potencial de representar sumideros de $\mathrm{C}$ y de nutrientes; sin embargo también pueden transformarse en fuentes de $\mathrm{C}$ de manera directa si son quemados o de manera indirecta si son removidos, situación que prevalece en la SNO. Las prácticas de aprovechamiento posteriores a la extracción son fundamentales para lograr un aprovechamiento sostenible de los bosques; por ello, los factores que influencian la cantidad, calidad y dinámica de los RLG deben ser identificados para planear mejor la manera de manejarlos.

\section{Conclusiones}

En el presente estudio se determinó la biomasa y necromasa de bosques con aprovechamiento forestal en el sur de México en función del régimen de precipitación. La contribución a la masa total de los diferentes componentes fue del 74, 4, 17 y $5 \%$ en la biomasa aérea, biomasa subterránea, RLG y mantillo, respectivamente. La respuesta de la biomasa y de la necromasa al aprovechamiento forestal fue dependiente de la cantidad anual de precipitación que recibe el sitio; por lo que no existe un efecto único de la extracción forestal sobre la masa de los distintos componentes. El aprovechamiento forestal influyó en la magnitud y en la distribución de la biomasa y de la necromasa en los diferentes componentes en los bosques a continuación del aprovechamiento (luego de un año de aprovechamiento); sin embargo se observó un proceso de recuperación de los diferentes componentes de este ecosistema a los 20 años. La madera muerta es un componente estructural masivo en los bosques bajo aprovechamiento, que puede representar hasta el $60 \%$ de la masa total de los bosques. La estrategia de aprovechamiento de los bosques en la SNO produjo incrementos en la magnitud de la necromasa. Los resultados de este esta investigación sugieren que los bosques templados de la SNO tienen un potencial importante de capturar $\mathrm{C}$ mediante la acumulación de biomasa aérea en los bosques jóvenes y la conservación de los RLG en el piso forestal; aspectos que deben ser considerados al proponer estrategias de mitigación al cambio climático en bosques templados de México.

\section{Agradecimientos}

Los autores de este trabajo agradecen a Ricardo López Martínez, María Luisa Cuevas Fernández, Manuel Canto Vergara, Rigoberto Romualdo Romualdo, Israel Cárdenas Camargo y Erika Balda Martínez Monzón por el apoyo en el trabajo de campo. Agradecemos al Comisario de Bienes Ejidales y a la comunidad de Ixtlán de Juárez, en la Sierra Norte de Oaxaca por permitirnos trabajar en sus bosques para la realización de este trabajo. Asimismo agradecemos al M. en C. Enrique Solís Villalpando por su apoyo en el trabajo de campo y laboratorio. Leopoldo Galicia reconoce el apoyo académico para esta investigación al proyecto IN 105712, DGAPA-PAPIIT. Finalmente, los autores agradecemos a dos revisores anónimos por sus comentarios y sugerencias que permitieron que el presente artículo mejorara considerablemente.

\section{Literatura citada}

Arriaga-Cabrera L., Aguilar V., Espinoza J.M., Galindo C., Herrmann H., Santana E., Graf-Montero S., Pisanty I. y Rosenzweig L. 2009. Regiones prioritarias y planeación para la conservación de la biodiversidad. En: CONABIO. Capital Natural de México, vol. II: Estado de Conservación y Tendencias de Cambio, pp. 433-457, Comisión Nacional para el Conocimiento y Uso de la Biodiversidad, México. 
Austin A.T. 2002. Differential effects of precipitation on production and decomposition along a rainfall gradient in Hawaii. Ecology 83:328-338.

Balboa-Murias M.A., Rodríguez-Soalleiro R., Merino A. y Álvarez-González J.G. 2006. Temporal variations and distribution of carbon stocks in aboveground biomass of radiata pine and maritime pine pure stands under different silvicultural alternatives. Forest Ecology and Management 237:29-38.

Bray D., Merino L. y Barry D. 2008. Los Bosques Comunitarios de México: Manejo Sustentable de Paisajes Forestales. Instituto Nacional de Ecología/Secretaría de Medio Ambiente y Recursos Naturales, México.

Berg B. y Laskowski R. 2006. Litter Decomposition: a Guide to Carbon and Nutrient Turnover. Elsevier, San Diego.

Brais S., Harvey B.D., Bergeron Y., Messier C., Greene D., Belleau A. y Paré D. 2004. Testing Forest ecosystem management in boreal mixedwoods in northweastern Quebec: initial response to aspen stands to different levels of harvesting. Canadian Journal of Forest Research 34:431-446.

Burton J.I., Ares A., Olson D.H. y Puettmann K.J. 2013. Management trade-off between aboveground carbon storage and understory plant species richness in temperate forests Ecological Applications 23:1297-1310.

Campo J., Gallardo J.F. y Hernández G. 2014. Leaf and litter nitrogen and phosphorus in three forests with low P supply. European Journal of Forest Research 133:121-129.

Cárdenas-Camargo I. 2008. Efecto de la calidad del tejido foliar en el proceso de descomposición en bosques de pino-encino bajo condiciones contrastantes de precipitación. Posgrado en Ciencias Biológicas, Maestría en Ciencias Biológicas (Biología Ambiental), Universidad Nacional Autónoma de México.

Carmona M.R., Armesto J.J., Aravena J.C. y Pérez C.A. 2002. Coarse woody debris biomass in successional and primary temperate forest in Chiloé Island, Chile. Forest Ecology and Management 164:265-275.

Currie W.S. y Nadelhoffer K.J. 2002. The Imprint of land use history: patterns of carbon and nitrogen in downed woody debris at the Harvard forest. Ecosystems 5:446-460.

Díaz-Franco R., Acosta-Mireles M., Carrillo-Aanzures F., Buendía-Rodríguez E., Flores-Ayala E. y Etchevers-Barra J.D. 2007. Determinación de ecuaciones alométricas para estimar biomasa y carbono en Pinus patula Schl. et Cham. Madera y Bosques 13:25-34.

FAO. 2006. Tendencias y Perspectivas del sector Forestal en América Latina y el Caribe. $w w w$ fao.org.

FAO. 2005 Evaluación de los Recursos Forestales Mundiales 2005. www.fao.org.

García E. 1998. Carta de climas de la República Mexicana. Escala 1:1000 000 en tres colecciones de 16 hojas c/u de Temperatura, Precipitación y Climas. Comisión Nacional para el Conocimiento y Uso de la Biodiversidad, México.

García-Gonzalo J., Peltola H., Briceño-Elizondo E. y Kellomaki S. 2007. Changed thinning regimes may increase carbon stock under climate change: A case study from a Finnishboreal forest. Climatic Change 81:431-454.

Gómez-Mendoza L., Vega-Peña E., Ramírez M.I., Palacio-Prieto J.L. y Galicia L. 2006. Projecting land use change processes in the Sierra Norte of Oaxaca, Mexico. Applied Geography 26:276-290.

Gough C.M., Seiler J.R., Wiseman P.E. y Maier C.A. 2005. Soil
$\mathrm{CO}_{2}$ efflux in loblolly pine (Pinus taeda L.) plantations on the Virginia Piedmont and South Carolina Coastal Plain over rotation-length chronosequence. Biogeochemistry 73:127-147.

Gough C.M., Vogel C.S., Harrold K.H., Georges K. y Curtis P.S. 2007. The legacy of harvest and fires on ecosystem carbon storage in a north temperate forest. Global Change Biology 13:1935-1949.

Gucinski H., Vance E. y Reiners W.A. 1995. Potential effects of global climate change. En: Smith W.K. and Hinckley T.M. Eds. Ecophisiology of Coniferous Forest, pp. 309-331, Academic Press, Nueva York.

Harmon M.E., Franklin J.F., Swanson F.J., Sollins P., Gregory S.V., Lattin J.D., Anderson N.H., Cline S.P., Aumen N.G., Sedell J.R., Lienkaemper G.W., Cromack K. y Cummings K.W. 1986. Ecology of coarse woody debris in temperate ecosystems. Advances in Ecological Research 15:133-302.

Harmon M.E., y Hua C. 1991. Coarse woody debris dynamics in two old-growth ecosystems. BioScience 41:604-610.

Harmon M.E., Krankina O.N., Yatskov M. y Matthews E. 2001. Predicting broad-scale stores of woody detritus from plot-level data. En: Lal R., Kimble J.M., Follett R.F. y Stewart B.A. Eds. Assessment Methods for Soil Carbon, pp. 533-552, Lewis Publishers, Boca Raton.

Houghton R.A. 2005. Aboveground forest biomass and the global carbon balance. Global Change Biology 11:945-958.

INEGI. 2000. Conjunto Nacional de Datos Vectoriales Edafológico escala 1:250 000 Serie II.

Janisch J.E. y Harmon M.E. 2002. Successional changes in live and dead wood carbon. Tree Physiology 22:77-89.

Kurz-Besson C, Coûteaux M.M., Berg B., Remacle J., Ribeiro C., Romanyà J. y Thiéry J.M. 2006. A climate response function explaining most of the variation of the forest floor needle mass and the needle decomposition in pine forests across Europe. Plant and Soil 285:97-114.

Laclau P. 2003. Root biomass and carbon storage of ponderosa pine in a northwest Patagonia plantation. Forest Ecology and Management 173:353-360.

Laiho R., Sanchez F., Tiarks A., Dougherty P.M. y Trettin C.C. 2003. Impacts of intensive forestry on early rotation trends in site carbon pools in the southeastern US. Forest Ecology and Management 174:177-189.

Leighty W.W., Hamburg S.P. y Caouette J. 2006. Effects of management on carbon sequestration in forest biomass in southeast Alaska. Ecosystems 9:1051-1065.

Lindner M. y Karjalainen T. 2007. Carbon inventory methods and carbon mitigation potentials of forest in Europe: a short review of recent progress. European Journal of Forest Research 126:149-156.

Llorente-Bousquets, J. y Ocegueda S. 2008. Estado del Conocimiento de la Biota, En: CONABIO 2008. Capital Natural de México, vol. I: Conocimiento Actual de la Biodiversidad, pp. 283-322, Comisión Nacional para el Conocimiento y Uso de la Biodiversidad, México.

Meier I.C. y Leuschner C. 2010.Variation of soil and biomass carbon pools in beech forests across a precipitation gradient. Global Change Biology 16:1035-1045.

Millennium Ecosystem Assessment Synthesis Report. 2005. Ecosystems and Human Well-being: Synthesis. Island Press, Washington.

Newman G.S. y Hart S.C. 2006. Nutrient covariance between fo- 
rest foliage and fine roots. Forest Ecology and Management 236:136-141.

Pan Y., Birdsey R.A., Fang J., Houghton R., Kauppi P.E., Kurz W.A., Phillips O.L., Shvidenko, A., Lewis S.L., Canadell J. G. Ciais P, Jackson R.B., Pacala S.W., McGuire A.D., Piao S., Rautiainen A., Sitch S. y Hayes, D. 2011. A large and persistent carbon sink in the world's forests. Science 333:988-992.

Park B.B., Yanai R.D., Fahey T.J., Bailey S.W., Siccama T.G., Shaley J.B. y Cleavitt N.L. 2008. Fine root dynamics and forest production across a calcium gradient in northern hardwood and conifer ecosystems. Ecosystems 11:325-341.

Peichl M. y Arain M.A. 2006. Above-and belowground ecosystem biomass and carbon pools in an age-sequence of temperate pine plantation forests. Agricultural Forest Meteorology 140:51-63.

Peichl M. y Arain M.A. 2007. Allometry and partitioning of above-and belowground tree biomass in an age-sequence of white pine forest. Forest Ecology and Management 253:68-80.

Porté A., Trichet P., Bert D. y Loustau D. 2002. Allometric relationship for branch and tree woody biomass of Maritime pine ( $\mathrm{Pi}$ nus pinaster Aït). Forest Ecology and Management 158:71-83.

Pregitzer K.S. y Eurskirchen E.S. 2004. Carbon cycling and storage in world forest: biome patterns related to forest age. Global Change Biology 10:2052-2077.

Pypker T.G. y Fredeen A.L. 2003. Belowground $\mathrm{CO}_{2}$ efflux from cut blocks of varying ages in sub-boreal British Columbia. Forest Ecology and Management 172:249-259.

Rubino D.L. y McCarthy B.C. 2003. Evaluation of coarse woody debris and forest vegetation across topographic gradients in a southeastern Ohio forest. Forest Ecology and Management 183:221-238.

Roxbourgh S.H., Wood S.W., Mackey B.G., Woldendorp G. y Gibbons P. 2006. Assessing the carbon sequestration potential of managed forest: a case study from temperate Australia. Journal of Applied Ecology 43:1149-1159.

Saynes V., Etchevers J.D., Galicia L., Hidalgo C. y Campo J. 2012. Soil carbon dynamics in high-elevation temperate forests of
Oaxaca (Mexico): thinning and rainfall effects. Bosque 33:3-11. Schlesinger W.H. 1997. Biogeochemistry: An Analysis of Global Change. Academic Press, San Diego.

SmartWood Program y Consejo Civil Mexicano para la Silvicultura Sostenible. 2001. Resumen Público de Certificación de la Comunidad Ixtlán de Juárez. SmartWood Program. < http:// www.rainforest-alliance.org/forestry/documents/comunidadixtlan.pdf $>$ (Consultado 8 agosto 2015).

Siry J.P., Cubbagy F.W. y Ahmed M.R. 2005. Sustainable Forest Management: global trends and opportunities. Forest Policy Economics 7:551-561.

Tang J., Qi Y., Xu M., Misson L. y Goldstein A.H. 2005. Forest thinning and soil respiration in a ponderosa pine plantation in the Sierra Nevada. Tree Physiology 25:57-66.

Tateno R., Hishi T. y Takeda H. 2004. Above-and belowground biomass and net primary production in a cool-temperate deciduous forest in relation to topographical changes in soil nitrogen. Forest Ecology and Management 193:297-306.

Tufekcioglu A., Raich J.W., Isenhart T.M. y Schultz R.C. 2003. Biomass, carbon and nitrogen dynamics of multi-species riparian buffers within an agricultural watershed in Iowa, USA. Agroforestry Systems 57:187-198.

White L.L., Zak D.R. y Barnes B.V. 2004. Biomass accumulation and soil nitrogen availability in an 87-year-old Populus gradidentata chronosequence. Forest Ecology and Management 191:121-127.

Xiao C.W. y Ceulemans R. 2004. Allometric relationship for below-and aboveground biomass of young Scot pines. Forest Ecology and Management 203:177-186.

Yin X., Perry J.A. y Dixon R.K. 1989. Fine-root dynamics and biomass distribution in a Quercus ecosystem following harvesting. Forest Ecology and Management 27:159-177.

Zhang D., Hui D., Luo Y. y Zhou G. 2008 Rates of litter decomposition in terrestrial ecosystems: global patterns and controlling factors. Journal Plant Ecology 1:1-9.

Recibido: 6 de marzo de 2014

Aceptado: 4 de julio de 2014 